\title{
Dual-Quasi Elliptic Planar Motion
}

\author{
Murat BEKAR
}

(Communicated by Yusuf YAYLI)

\begin{abstract}
Dual-quaternions are an elegant and useful mathematical tools for representing rigid-body (screw) motions in three-dimensional Euclidean space $\mathbb{R}^{3}$. The aim of this paper is to consider the algebra of dual semi-quaternions with their basic properties and generalize the results of the Euclidean-planar motion given by Blaschke and Grünwald to dual planar motion.
\end{abstract}

Keywords: Dual-numbers, Dual Semi-Quaternions, Quasi-Elliptic Motion.

AMS Subject Classification (2010): 11E88; 11R52; 35H30; 53A17; 53A20; 53A35.

\section{INTRODUCTION}

Quaternions are discovered by Sir William R. Hamilton in the middle of $19^{\text {th }}$ century. He want to construct an algebraic system whose elements comprise of a real and two imaginary part. The predicament in the development of this algebraic system occured by defining the mulitiplication rule. He overcame with this problem by using the three imaginary part $i, j, k$ with the multiplication rules

$$
i^{2}=j^{2}=k^{2}=i j k=-1
$$

and called the real space spanned by the elements $1, i, j, k$ as quaternions, see [1].

Quaternions have some applications in physics, kinematics, mechanism, computer simulations, etc. For example, rotations in three-dimensional Euclidean space $\mathbb{R}^{3}$ can be represented by real-quaternions, while rigid-body motions in $\mathbb{R}^{3}$ can be represent by dual-quaternions, see [2-4]. Also, quasi-elliptic motions obtained by the kinematic mapping of Blaschke and Grünwald can be represented by semi-quaternions, see [5, 6]. In this paper, firstly a brief summary of the concepts dual-projective plane, dual quasi-elliptic geometry and dual semi-quaternions are given. Afterwards, the results of the Euclidean-planar motion given by Blaschke and Grünwald are generalized to dual planar-motion.

\section{PRELIMINARIES}

In this section, an overview of each of the concepts dual-projective plane, dual-quasi elliptic geometry and dual semi-quaternions is given.

\subsection{Dual-Projective Plane}

The set of dual-numbers is defined to be

$$
\mathbb{D}=\left\{A=a+\varepsilon a^{*}:\left(a, a^{*}\right) \in \mathbb{R}^{2}, \varepsilon \neq 0 \text { and } \varepsilon^{2}=0\right\}
$$

Received : 28-January-2016, Accepted : 11-April-2016 
where $\varepsilon$ is the dual unit and commutes with real-numbers that is $r \varepsilon=\varepsilon r$ for all $r \in \mathbb{R}$. Also, the real-numbers $a$ and $a^{*}$ are called the non-dual and the dual parts of $A$, respectively. If $a=0$ (resp. $a \neq 0$ ), then $A$ is said to be a pure (resp. non-pure). The set of all pure (resp. non-pure) dual-numbers is denoted by $\mathbf{D}$ (resp. $\breve{\mathbb{D}}$ ).

Let $A=a+\varepsilon a^{*}$ and $B=b+\varepsilon b^{*}$ be any two dual-numbers. Then,

- The addition of $A$ and $B$ is

$$
A+B=(a+b)+\varepsilon\left(a^{*}+b^{*}\right) .
$$

- The multiplication (known as the Study multiplication) of $A$ and $B$ is

$$
A B=B A=(a b)+\varepsilon\left(a b^{*}+b a^{*}\right) .
$$

- The equality of $A$ and $B$ is defined to be

$$
A=B \quad \text { iff } \quad a=b \quad \text { and } \quad a^{*}=b^{*} .
$$

- The dual conjugate of $A$ is defined to be

$$
A^{\star}=a-\varepsilon a^{*} .
$$

- The square root of $A=a+\varepsilon a^{*}$ exists only for $a>0$ and is defined to be

$$
\sqrt{A}=\sqrt{a}+\varepsilon \frac{a^{*}}{2 \sqrt{a}} .
$$

- The norm of $A$ is defined to be

$$
N_{A}=A A^{\star}=A^{\star} A=a^{2},
$$

while its modulus is defined to be

$$
\|A\|=\sqrt{N_{A}}=|a|
$$

where $|$,$| denotes the absolute value of a real-number. If N_{A}=\|A\|=1$ (that is, $A= \pm 1+\varepsilon a^{*}$ ), then $A$ is said to be a unit.

- The multiplicative inverse of a dual-number is obtained by dividing its dual-conjugate by its norm. It is important to emphasize that the multiplicative inverse of a dual-number exists only if it is a non-pure one. For example, the multiplicative inverse of $A$ exists only for $a \neq 0$ and is defined to be

$$
A^{-1}=\frac{A^{\star}}{N_{A}}=\frac{1}{a}-\varepsilon \frac{a^{*}}{a^{2}} .
$$

The dual angle $\Theta=\theta+\varepsilon \theta^{*} \in \mathbb{D}$ represents the relative displacement and orientation between any two lines $l_{1}, l_{2}$ in space $\mathbb{R}^{3}$, see Fg. 1, where

$$
\begin{gathered}
\theta \in \mathbb{R} \text { is the projected angle between the lines } l_{1} \text { and } l_{2}, \\
\theta^{*} \in \mathbb{R} \text { is the shortest distance between the lines } l_{1} \text { and } l_{2} .
\end{gathered}
$$

The trigonometric functions sine, cosine and tangent of a dual angle $\Theta=\theta+\varepsilon \theta^{*}$ are defined to be

$$
\begin{gathered}
\sin \Theta=\sin \theta+\varepsilon \theta^{*} \cos \theta \\
\cos \Theta=\cos \theta-\varepsilon \theta^{*} \sin \theta \\
\tan \Theta=\tan \theta+\varepsilon \theta^{*} \sec ^{2} \theta
\end{gathered}
$$




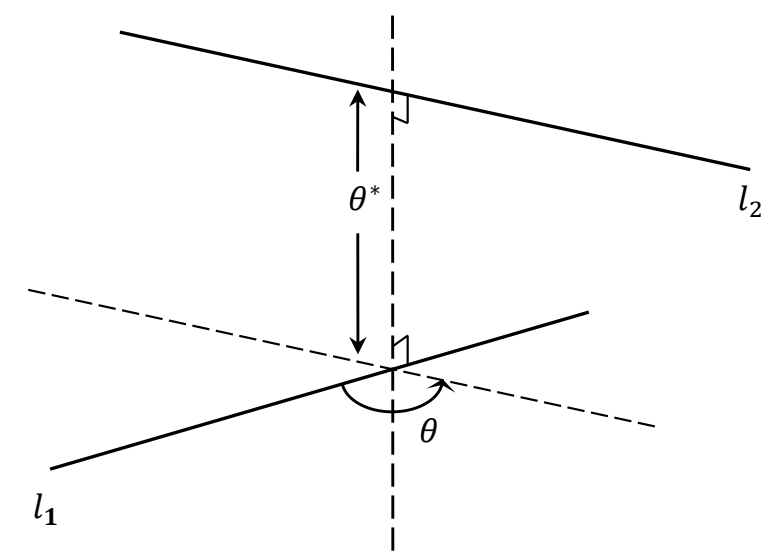

Figure 1. The dual angle $\Theta=\theta+\varepsilon \theta^{*} \in \mathbb{D}$ expresses the relationship between the $l_{1}$ and $l_{2}$ in $\mathbb{R}^{3}$.

For further information about dual-numbers see $[7,8]$.

The set

$$
\mathbb{D}^{2}=\{\widetilde{A}=(X, Y): X, Y \in \mathbb{D}\}
$$

is a two-dimensional module over the ring $\mathbb{D}$ and is called the dual-plane. The elements of $\mathbb{D}^{2}$ are also called the dual-vectors in $\mathbb{D}^{2}$. A dual-vector $\widetilde{A}=(X, Y)$ can be written in dual form as

$$
\widetilde{A}=\vec{z}+\varepsilon \overrightarrow{z^{*}}
$$

where $X=x+\varepsilon x^{*}, Y=y+\varepsilon y^{*} \in \mathbb{D}$ and $\vec{z}=(x, y), \overrightarrow{z^{*}}=\left(x^{*}, y^{*}\right) \in \mathbb{R}^{2}$.

The set

$$
\mathbb{D}^{3}=\{\widehat{A}=(X, Y, Z): X, Y, Z \in \mathbb{D}\}
$$

is a three-dimensional module over the ring $\mathbb{D}$ and is called the dual-space (or $\mathbb{D}$ - module). The elements of $\mathbb{D}^{3}$ are also called the dual-vectors in $\mathbb{D}^{3}$. A dual-vector $\widehat{A}=(X, Y, Z)$ can be written in dual form as

$$
\widehat{A}=\overrightarrow{\boldsymbol{w}}+\varepsilon \overrightarrow{\boldsymbol{w}^{*}}
$$

where

$$
X=x+\varepsilon x^{*}, Y=y+\varepsilon y^{*}, Z=z+\varepsilon z^{*} \in \mathbb{D}
$$

and

$$
\overrightarrow{\boldsymbol{w}}=(x, y, z), \overrightarrow{\boldsymbol{w}^{*}}=\left(x^{*}, y^{*}, z^{*}\right) \in \mathbb{R}^{3} .
$$

Let

$$
\widehat{A}=(X, Y, Z)=\overrightarrow{\boldsymbol{w}}+\varepsilon \overrightarrow{\boldsymbol{w}^{*}}, \widehat{B}=(P, Q, R)=\overrightarrow{\boldsymbol{v}}+\varepsilon \overrightarrow{\boldsymbol{v}}
$$

be any two dual-vectors where

$$
\begin{gathered}
X=x+\varepsilon x^{*}, Y=y+\varepsilon y^{*}, \quad Z=z+\varepsilon z^{*} \in \mathbb{D} \\
P=p+\varepsilon p^{*}, Q=q+\varepsilon q^{*}, \quad R=r+\varepsilon r^{*} \in \mathbb{D}
\end{gathered}
$$

and

$$
\begin{aligned}
\overrightarrow{\boldsymbol{w}} & =(x, y, z), \overrightarrow{\boldsymbol{w}^{*}}=\left(x^{*}, y^{*}, z^{*}\right) \in \mathbb{R}^{3} \\
\overrightarrow{\boldsymbol{v}} & =(p, q, r), \overrightarrow{\boldsymbol{v}^{*}}=\left(p^{*}, q^{*}, r^{*}\right) \in \mathbb{R}^{3} .
\end{aligned}
$$

Then, 
1. The addition of $\widehat{A}=(X, Y, Z)=\overrightarrow{\boldsymbol{w}}+\varepsilon \overrightarrow{\boldsymbol{w}^{*}}$ and $\widehat{B}=(P, Q, R)=\overrightarrow{\boldsymbol{v}}+\varepsilon \overrightarrow{\boldsymbol{v}^{*}}$ is

$$
\begin{aligned}
\widehat{A}+\widehat{B} & =(X+P, Y+Q, Z+R) \\
& =(\overrightarrow{\boldsymbol{w}}+\overrightarrow{\boldsymbol{v}})+\varepsilon\left(\overrightarrow{\boldsymbol{w}^{*}}+\overrightarrow{\boldsymbol{v}^{*}}\right)
\end{aligned}
$$

2. The inner product of $\widehat{A}=(X, Y, Z)=\overrightarrow{\boldsymbol{w}}+\varepsilon \overrightarrow{\boldsymbol{w}} \overrightarrow{\boldsymbol{w}}^{*}$ and $\widehat{B}=(P, Q, R)=\overrightarrow{\boldsymbol{v}}+\varepsilon \overrightarrow{\boldsymbol{v}^{*}}$ is

$$
\begin{aligned}
<\widehat{A}, \widehat{B}>_{d} & =X P+Y Q+Z R \\
& =\langle\overrightarrow{\boldsymbol{w}}, \overrightarrow{\boldsymbol{v}}\rangle+\varepsilon\left(\left\langle\overrightarrow{\boldsymbol{w}}, \overrightarrow{\boldsymbol{v}^{*}}\right\rangle+\left\langle\overrightarrow{\boldsymbol{w}^{*}}, \overrightarrow{\boldsymbol{v}}\right\rangle\right)
\end{aligned}
$$

where "<, > denotes the usual inner-product in $\mathbb{R}^{3}$. If $<\widehat{A}, \widehat{B}>_{d}=0$, then $\widehat{A}$ and $\widehat{B}$ are said to be perpendicular in the sense of dual.

3. The vector product of $\widehat{A}=(X, Y, Z)=\overrightarrow{\boldsymbol{w}}+\varepsilon \overrightarrow{\boldsymbol{w}^{*}}$ and $\widehat{B}=(P, Q, R)=\overrightarrow{\boldsymbol{v}}+\varepsilon \overrightarrow{\boldsymbol{v}^{*}}$ is

$$
\begin{aligned}
\widehat{A} \times{ }_{d} \widehat{B} & =(Y R-Q Z, Z P-R X, X Q-P Y) \\
& =\overrightarrow{\boldsymbol{w}} \times \overrightarrow{\boldsymbol{v}}+\varepsilon\left(\overrightarrow{\boldsymbol{w}} \times \overrightarrow{\boldsymbol{v}^{*}}+\overrightarrow{\boldsymbol{w}^{*}} \times \overrightarrow{\boldsymbol{v}}\right)
\end{aligned}
$$

where " $\times$ " denotes the usual vector-product in $\mathbb{R}^{3}$.

For further information about dual-plane and dual-space see $[7,9,10]$.

Let $\widehat{P_{0}}=\left(X_{0}, Y_{0}, Z_{0}\right)$ be a point and $\widehat{N}=(A, B, C)$ be a non-zero vector in $\mathbb{D}^{3}$. The plane passes through $\widehat{P_{0}}$ and perpendicular to $\widehat{N}$ consists of all the points $\widehat{P}=(X, Y, Z) \in \mathbb{D}^{3}$ such that the vector from $\widehat{P_{0}}$ to $\widehat{P}$ (that is, $\left.\widehat{P_{0}} \widehat{P}\right)$ is perpendicular to the vector $\widehat{N}$, see Fig. 2. In other words,

$$
<\widehat{\mathbf{N}}, \overrightarrow{\widehat{P_{0}}} \stackrel{\widehat{P}}{>_{d}}=A\left(X-X_{0}\right)+B\left(Y-Y_{0}\right)+C\left(Z-Z_{0}\right)=0 .
$$

Thus, the plane through $\widehat{P_{0}}$ and perpendicular to $\widehat{N}$ has the equation (in general form)

$$
A X+B Y+C Z=D
$$

where $D=A X_{0}+B Y_{0}+C Z_{0}$. For the special case $D=0$, the plane passes through the origin.

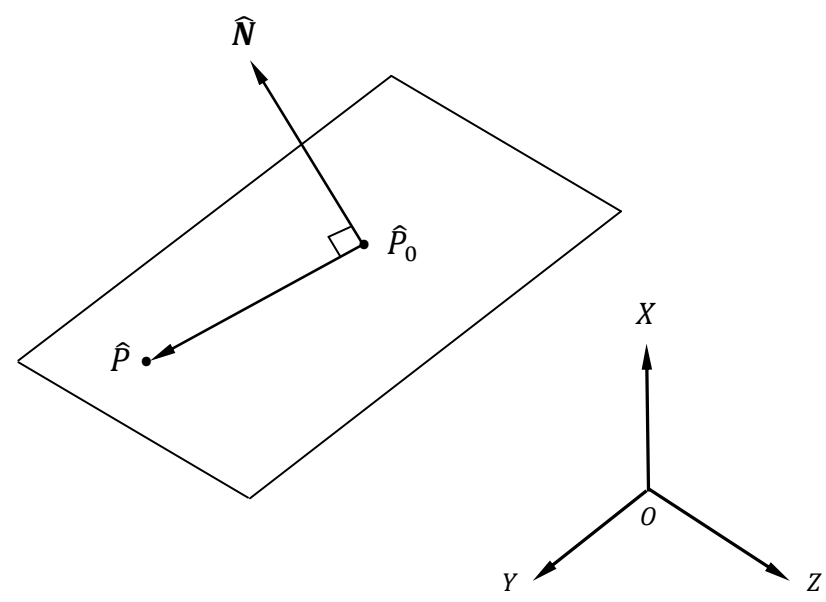

Figure 2. The dual-plane passing through the point $\widehat{P_{0}}$ and perpendicular to the vector $\widehat{N}$ in $\mathbb{D}^{3}$

Let the linear space $\mathbb{D}^{3}$ be equipped with the coordinates $X, Y, Z$. The set of the one-dimensional subspaces of $\mathbb{D}^{3}$ is called the dual-projective plane and is denoted by $\mathbb{P}^{2}$. A two-dimensional dual-plane $\mathbb{D}^{2}$ can be embedded into $\mathbb{P}^{2}$ by

$$
\widetilde{A}=(Y, Z) \longmapsto \widehat{A} \mathbb{D}=(1, Y, Z) \mathbb{D}=(\lambda, \lambda Y, \lambda Z), \lambda \in \mathbb{D} .
$$




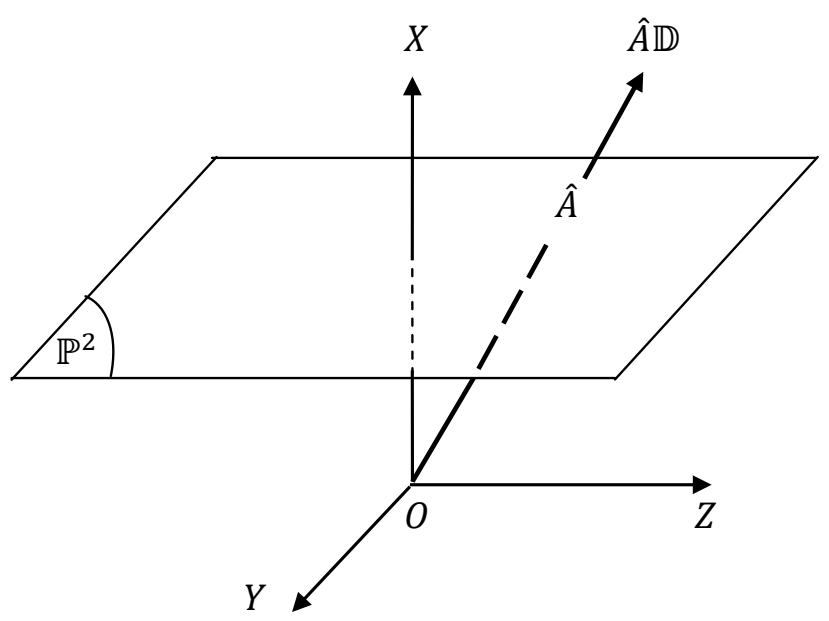

Figure 3. Dual-projective plane $\mathbb{P}^{2}$ where $\widehat{A}=(1, Y, Z) \in \mathbb{P}^{2}$.

For the special case $X=0$, the point $\widehat{A} \mathbb{D}=(X, Y, Z) \mathbb{D}$ is called the ideal point, see Fig. 3.

If $\widehat{A} \mathbb{D}=(X, Y, Z) \mathbb{D}$ is a proper point (that is $X \in \breve{\mathbb{D}})$, its coordinates are recovered in $\mathbb{D}^{2}$ by

$$
(X, Y, Z) \mathbb{D} \in \mathbb{P}^{2} \Longleftrightarrow(Y / X, Z / X) \in \mathbb{D}^{2} .
$$

If a line $L$ of $\mathbb{D}^{2}$ is parallel to the vector $\left(L_{1}, L_{2}\right)$, then it has the ideal point $\left(0, L_{1}, L_{2}\right) \mathbb{D}$. The plane $X=0$ corresponds to the ideal (or absolute) line $W$ which contains all the ideal points. The homogeneous coordinate vector of the point $\widehat{A} \mathbb{D}$ is the coordinate vector $\widehat{A}$ which is represented as column vector in matrix notation.

\subsection{Dual-Quasi Elliptic Geometry}

Suppose that $L$ is a non-horizontal line in $\mathbb{D}^{3}$ intersecting the dual-planes $Z=0, Z=+1, Z=-1$, respectively, at the points $M, L_{1}, L_{2}$. And suppose that $L_{1}^{\prime}, L_{2}^{\prime}$ are the normal piercing points, respectively, of the points $L_{1}, L_{2}$ on the plane $Z=0$. Moreover, let $\beta^{-}, \beta^{+}$be two mappings that rotates, respectively, the points $L_{2}^{\prime}, L_{1}^{\prime}$ in the plane $Z=0$ around the point $M$ (which is the midpoint of the points $L_{1}^{\prime}, L_{2}^{\prime}$ ) with a positive oriented hyperbolic right angle as

$$
L_{2}^{\prime} \beta^{-}=L_{2}^{\prime \prime}, \quad L_{1}^{\prime} \beta^{+}=L_{1}^{\prime \prime},
$$

see Fig. 4 . In this case, $\beta^{-}$and $\beta^{+}$are linear mappings from the dual-space of lines onto the horizontal dual-plane $Z=0$, and there exists an ordered point pair $L_{1}^{\prime \prime}, L_{2}^{\prime \prime}$ associated with every ideal line $W$. That means, if we choose an ordered pair of dual in the plane $Z=0$, then there exists a unique line $L$ associated with them. Also, there exists an invertible relationship $L \leftrightarrow\left(L_{1}^{\prime \prime}, L_{2}^{\prime \prime}\right)$ between the non-horizontal lines $L$ of $\mathbb{D}^{3}$ and the ordered pairs $\left(L_{1}^{\prime \prime}, L_{2}^{\prime \prime}\right)$ of dual.

\subsection{Dual Semi-Quaternions}

A dual semi-quaternion is defined by

$$
Q=Q_{0}+Q_{1} i+Q_{2} j+Q_{3} k
$$

where $Q_{i}=q_{i}+\varepsilon q_{i}^{*}$ are dual-numbers for $i=0,1,2,3$. Also, $1, \boldsymbol{i}, \boldsymbol{j}, \boldsymbol{k}$ may be interpreted as the four basic vectors of Cartesian set of coordinates satisfying the following non-commutative multiplication rules

$$
\begin{gathered}
\boldsymbol{i}^{2}=-1, \boldsymbol{j}^{2}=\boldsymbol{k}^{2}=0, \\
\boldsymbol{i} \boldsymbol{j}=-\boldsymbol{j} \boldsymbol{i}=\boldsymbol{k}, \quad \boldsymbol{j} \boldsymbol{k}=-\boldsymbol{k} \boldsymbol{j}=0, \quad \boldsymbol{k} \boldsymbol{i}=-\boldsymbol{i} \boldsymbol{k}=\boldsymbol{j} .
\end{gathered}
$$

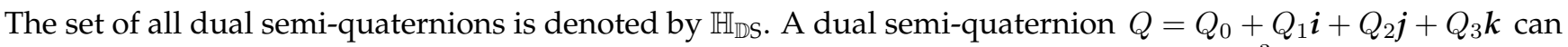
be written as a sum of a scalar part $S_{Q}=Q_{0} \in \mathbb{D}$ and vector part $\boldsymbol{V}_{Q}=Q_{1} \boldsymbol{i}+Q_{2} \boldsymbol{j}+Q_{3} \boldsymbol{k} \in \mathbb{D}^{3}$ that is $Q=S_{Q}+\boldsymbol{V}_{Q}$. If $S_{Q}=0$, then $Q$ is said to be a pure and is denoted by boldface letter $Q$. The set of all pure dual-quaternions is 


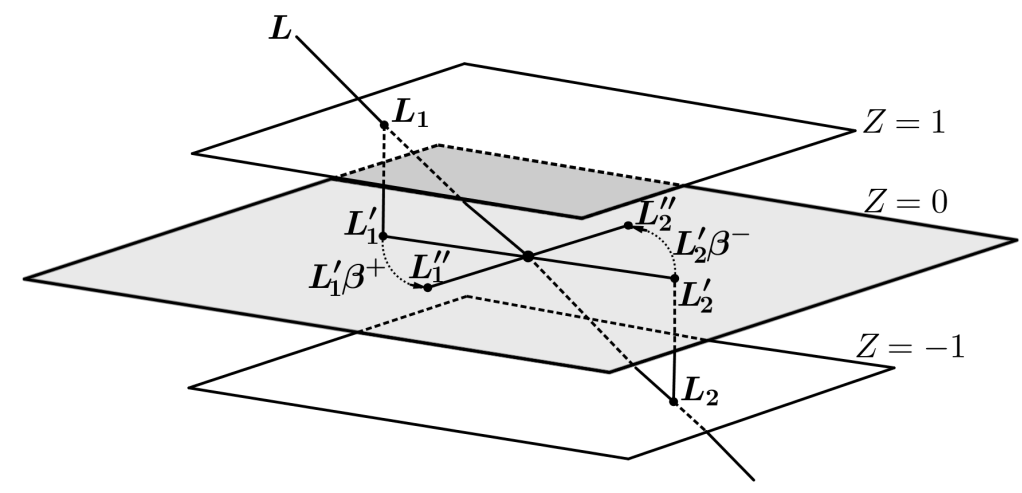

Figure 4. Dual-quasi elliptic motion.

denoted by $\mathbf{H}_{\mathrm{DS}}$.

Let $Q=S_{Q}+\boldsymbol{V}_{Q}=Q_{0}+Q_{1} \boldsymbol{i}+Q_{2} \boldsymbol{j}+Q_{3} \boldsymbol{k}$ and $P=S_{P}+\boldsymbol{V}_{P}=P_{0}+P_{1} \boldsymbol{i}+P_{2} \boldsymbol{j}+P_{3} \boldsymbol{k}$ be any two dual semi-quaternions. Then,

- The addition of $Q$ and $P$ is

$$
\begin{aligned}
Q+P & =\left(S_{Q}+S_{P}\right)+\left(\boldsymbol{V}_{Q}+\boldsymbol{V}_{P}\right) \\
& =\left(Q_{0}+P_{0}\right)+\left(Q_{1}+P_{1}\right) \boldsymbol{i}+\left(Q_{2}+P_{2}\right) \boldsymbol{j}+\left(Q_{3}+P_{3}\right) \boldsymbol{k}
\end{aligned}
$$

- The multiplication of $Q$ and $P$ is

$$
\begin{aligned}
Q P= & S_{Q} S_{P}-<\boldsymbol{V}_{Q}, \boldsymbol{V}_{P}>_{d}^{\prime}+S_{Q} \boldsymbol{V}_{P}+S_{P} \boldsymbol{V}_{Q}+\boldsymbol{V}_{Q} \times_{d}^{\prime} \boldsymbol{V}_{P} \\
= & \left(Q_{0} P_{0}-Q_{1} P_{1}\right)+\left(Q_{1} P_{0}+Q_{0} P_{1}\right) \boldsymbol{i}+ \\
& \left(Q_{2} P_{0}+Q_{3} P_{1}+Q_{0} P_{2}-Q_{1} P_{3}\right) \boldsymbol{j}+ \\
& \left(Q_{0} P_{3}+Q_{1} P_{2}+Q_{3} P_{0}-Q_{2} P_{1}\right) \boldsymbol{k}
\end{aligned}
$$

where $<\boldsymbol{V}_{Q}, \boldsymbol{V}_{P}>_{d}^{\prime}=Q_{1} P_{1}$ and $\boldsymbol{V}_{Q} \times_{d}^{\prime} \boldsymbol{V}_{P}=0 \boldsymbol{i}+\left(Q_{3} P_{1}-Q_{1} P_{3}\right) \boldsymbol{j}+\left(Q_{1} P_{2}-Q_{2} P_{1}\right) \boldsymbol{k}$.

- The equality of $Q$ and $P$ is defined to be

$$
Q=P \quad \text { iff } \quad S_{Q}=S_{P} \text { and } \quad V_{Q}=V_{P} .
$$

- The quaternionic conjugate of $Q$ is defined to be

$$
\bar{Q}=S_{Q}-\boldsymbol{V}_{Q}=Q_{0}-Q_{1} \boldsymbol{i}-Q_{2} \boldsymbol{j}-Q_{3} \boldsymbol{k},
$$

while its dual conjugate is defined to be

$$
Q^{\star}=S_{Q}^{\star}+V_{Q}^{\star}=Q_{0}^{\star}+Q_{1}^{\star} i+Q_{2}^{\star} j+Q_{3}^{\star} k .
$$

- The norm of $Q$ is defined to be

$$
N_{Q}=Q \bar{Q}=\bar{Q} Q=Q_{0}^{2}+Q_{1}^{2}=\left(q_{0}^{2}+q_{1}^{2}\right)+2 \varepsilon\left(q_{0} q_{0}^{*}+q_{1} q_{1}^{*}\right),
$$

while its modulus is defined to be

$$
\|Q\|=\sqrt{N_{Q}}=\sqrt{q_{0}^{2}+q_{1}^{2}}+\varepsilon \frac{q_{0} q_{0}^{*}+q_{1} q_{1}^{*}}{\sqrt{q_{0}^{2}+q_{1}^{2}}}
$$

for $q_{0}^{2}+q_{1}^{2} \neq 0$. If $N_{Q}=\|Q\|=1$, then $Q$ is said to be a unit. 
- The multiplicative inverse of a dual semi-quaternion is obtained by dividing its quaternionic-conjugate by its norm. It is important to emphasize that the multiplicative inverse of a dual semi-quaternion exists only if its norm is non-zero. For example, the multiplicative inverse of $Q$ exists only for $q_{0} \neq 0 \neq q_{1}$ and is defined to be

$$
Q^{-1}=\frac{\bar{Q}}{N_{Q}}
$$

The matrix representation of a dual semi-quaternion $Q=Q_{0}+Q_{1} i+Q_{2} j+Q_{3} k$ can be given by

$$
Q:=\mathrm{Q}=\left(\begin{array}{cccc}
Q_{0} & -Q_{1} & 0 & 0 \\
Q_{1} & Q_{0} & 0 & 0 \\
Q_{2} & Q_{3} & Q_{0} & -Q_{1} \\
Q_{3} & -Q_{2} & Q_{1} & Q_{0}
\end{array}\right)
$$

For the special case, if $Q$ is unit then $Q$ can be given in general form as

$$
\left(\begin{array}{cccc}
\cos \Theta & -\sin \Theta & 0 & 0 \\
\sin \Theta & \cos \Theta & 0 & 0 \\
Q_{2} & Q_{3} & \cos \Theta & -\sin \Theta \\
Q_{3} & -Q_{2} & \sin \Theta & \cos \Theta
\end{array}\right)
$$

where $\Theta=\theta+\varepsilon \theta^{*} \in \mathbb{D}$. It can be easily checked that $\mathrm{Q}$ is orthogonal because $\mathrm{I}=\mathrm{Q}^{\mathrm{T}} \mathrm{IQ}$ and $\operatorname{det} \mathrm{Q}=1$ for the metric tensor

$$
I=\left(\begin{array}{llll}
1 & 0 & 0 & 0 \\
0 & 1 & 0 & 0 \\
0 & 0 & 0 & 0 \\
0 & 0 & 0 & 0
\end{array}\right)
$$

where $\mathrm{Q}^{\mathrm{T}}$ denotes the transpose of $\mathrm{Q}$. Hence, unit dual semi-quaternions can be used to represent rotations.

The algebra $\mathbb{H}_{\mathbb{D S}}$ is isomorphic to the Clifford algebra $C l_{0,1,3}$ (i.e., $\mathbb{H}_{\mathbb{D S}} \cong C l_{0,1,3}$ ) in dimension 4 when we identify the quaternionic units $\boldsymbol{i}, \boldsymbol{j}, \boldsymbol{k}$, respectively, with $e_{1}, e_{2}, e_{12}\left(=e_{1} e_{2}\right)$, and the dual unit $\varepsilon$ with $e_{34}\left(=e_{3} e_{4}\right)$ which commutes with a subalgebra of $C l_{0,1,3}$ generated by $e_{1}$ and $e_{2}$. Here, the standard anti-commuting generators $e_{i}, i=1,2,3,4$, satisfy

$$
e_{1}^{2}=-1, e_{2}^{2}=e_{3}^{2}=e_{4}^{2}=0 \text { and } e_{i} e_{j}=-e_{j} e_{i} \text { for } i \neq j .
$$

\section{DUAL-QUASI ELLIPTIC MOTION IN $\mathbb{D}^{3}$}

Let $Q=Q_{0}+Q_{1} i+Q_{2} j+Q_{3} k$ be a unit dual semi-quaternion and $Q_{1}$ be a non-pure dual-number, i.e., $Q_{1} \in \breve{\mathbb{D}}$. Then the map

$$
\beta_{Q}: Q \mathbb{D} \in \mathbb{P}^{2} \mapsto\left(\begin{array}{ccc}
1 & 0 & 0 \\
2\left(Q_{1} Q_{2}+Q_{0} Q_{3}\right) & Q_{0}^{2}-Q_{1}^{2} & -2 Q_{0} Q_{1} \\
2\left(Q_{1} Q_{3}-Q_{0} Q_{2}\right) & 2 Q_{0} Q_{1} & Q_{0}^{2}-Q_{1}^{2}
\end{array}\right)=\mathrm{A}
$$

corresponds to each point a negative oriented rotation in the dual-projective plane $\mathbb{P}^{2}$. That is because the matrix $\mathrm{A}$ is orthogonal (i.e., $\mathrm{I}=\mathrm{A}^{\mathrm{T}} \mathrm{I} \mathrm{A}$ ) with $\operatorname{det} \mathrm{A}=1$.

Proposition 3.1. The unit dual semi-quaternion

$$
Q=-\cos \frac{\Theta}{2}+\sin \frac{\Theta}{2} i+Q_{2} j+Q_{3} k
$$

represents a negative oriented rotation in dual-plane $\mathbb{D}^{2}$, where $\sin (\Theta / 2)$ is a non-pure dual-number, i.e., $\sin (\Theta / 2) \in \breve{\mathbb{D}}$.

Proof. If we take $Q_{0}=-\cos (\Theta / 2), Q_{1}=\sin (\Theta / 2)$ then the map

$$
f_{Q}:\left(\begin{array}{c}
X \\
Y
\end{array}\right) \mapsto\left(\begin{array}{cc}
Q_{0}^{2}-Q_{1}^{2} & -2 Q_{0} Q_{1} \\
2 Q_{0} Q_{1} & Q_{0}^{2}-Q_{1}^{2}
\end{array}\right)\left(\begin{array}{c}
X \\
Y
\end{array}\right)+\left(\begin{array}{c}
2\left(Q_{1} Q_{2}+Q_{0} Q_{3}\right) \\
2\left(Q_{1} Q_{3}-Q_{0} Q_{2}\right)
\end{array}\right)
$$


represents a dual-planar motion. Also, it is straightforward to show that

$$
f_{Q}\left(\frac{Q_{2}}{\sin \frac{\Theta}{2}}, \frac{Q_{3}}{\sin \frac{\Theta}{2}}\right)=\left(\frac{Q_{2}}{\sin \frac{\Theta}{2}}, \frac{Q_{3}}{\sin \frac{\Theta}{2}}\right) .
$$

Thus, the linear map $f_{Q}$ represents a negative oriented rotation through an angle $\Theta \in \mathbb{D}$ about the center

$$
M=\left(\frac{Q_{2}}{\sin \frac{\Theta}{2}}, \frac{Q_{3}}{\sin \frac{\Theta}{2}}\right) \in \mathbb{D}^{2}
$$

in dual-plane $\mathbb{D}^{2}$.

Corollary 3.1. If a non-horizontal line $L$ is incident with a point $P \in \mathbb{P}^{2}$, then the rotation corresponding to $P$ maps $L_{2}^{\prime \prime}$ to $L_{1}^{\prime \prime}$ as in Fig. 4.

\section{References}

[1] WARD, J. P., Quaternions and Cayley Algebrs and Applications, Kluwer Academic Publishers, Dordrecht, 1996.

[2] Bekar, M. and Yayli, Y., Involutions of Complexified Quaternions and Split Quaternions, Advances in Applied Clifford Algebras (2013), 23, 283-299.

[3] Bekar, M. and Yayli, Y., Dual-quaternion Involutions and Anti-Involutions, Advances in Applied Clifford Algebras (2013), 23, 577-592.

[4] Ell, T. A. and Sangwine, S. J., Quaternion Involutions and Anti-involutions, Computers and Mathematics with Applications (2007), 53, 137-143.

[5] Blaschke, W., Euclidian kinematics and non-Euclidian geometry I, II, Zeitschrift für Mathematik und Physik (1911), 60, 61-92.

[6] Pottman, H., and Wallner, J., Computational Line Geometry, Springer-Verlag, Heidelberg, 2001.

[7] Blaschke, W., Differential Geometrie and Geometrischke Grundlagen ven Einsteins Relativitasttheorie, Dover, New York, 1945.

[8] Fischer, I. S., Dual-Number Methods in Kinematics, Statics and Dynamics. Boca Raton London New York Washington D.C.: CRC Press, 1999.

[9] Hacisalihoglu, H.H., Hareket Geometrisi ve Kuaterniyonlar Teorisi, Gazi Universitesi Fen-Edb. Fakultesi, 1983.

[10] Veldkamp, G.R., On the use of dual numbers, vectors and matrices in instantaneous spatial kinematics, Mech. Mach. Theory (1976), 11(2), 141-156.

99

\section{Affiliations}

\section{MURAT BEKAR}

ADDRESS: Necmettin Erbakan University, Department of Mathematics and Computer Sciences, 42090, KonyaTURKEY.

E-MAIL: mbekar@konya.edu.tr 